\title{
Isolation and characterization of adenoviruses infecting endangered golden snub-nosed monkeys (Rhinopithecus roxellana)
}

Bing Tan ${ }^{1,3}$, Li-Jun Wu' ${ }^{1}$ Xing-Lou Yang ${ }^{1}$, Bei Li', Wei Zhang ${ }^{1}$, Yong-Song Lei ${ }^{2}$, Yong Li ${ }^{2}$, Guo-Xiang Yang ${ }^{2}$, Jing Chen ${ }^{2}$, Guang Chen ${ }^{2}$, Han-Zhong Wang ${ }^{1}$ and Zheng-Li Shi ${ }^{1,3,4^{*}}$ [D

\begin{abstract}
Background: Adenoviruses are important pathogens with the potential for interspecies transmission between humans and non-human primates. Although many adenoviruses have been identified in monkeys, the knowledge of these viruses from the Colobinae members is quite limited.

Findings: We conducted a surveillance of viral infection in endangered golden snub-nosed monkeys (Rhinopithecus roxellana) in the subfamily Colobinae in China, and found that $5.1 \%$ of sampled individuals were positive for adenovirus. One of the adenoviruses (SAdV-WIV19) was successfully isolated and its full-length genome was sequenced. The full-length genome of WIV19 is 33,562 bp in size, has a $\mathrm{G}+\mathrm{C}$ content of $56.2 \%$, and encodes 35 putative genes. Sequence analysis revealed that this virus represents a novel species in the genus Mastadenovirus. Diverse cell lines, including those of human origin, were susceptible to WIV19.

Conclusion: We report the first time the isolation and full-length genomic characterization of an adenovirus from the subfamily Colobinae.
\end{abstract}

Keywords: Mastadenovirus, Simian adenovirus, Golden snub-nosed monkey

\section{Body of text}

Within the family Adenoviridae, the genus Mastadenovirus contains a group of non-enveloped icosahedral viruses that range in size from 70 to $90 \mathrm{~nm}$ and contain a linear double-stranded DNA genome of approximate $35 \mathrm{~kb}$ [1]. Members of this genus are pathogens that infect a wide range of mammals. In humans, adenoviruses (AdVs) cause a variety of pathologies including acute respiratory illness, epidemic keratoconjunctivitis, acute haemorrhagic cystitis, hepatitis, myocarditis, and gastroenteritis [2]. Although usually self-limiting, AdV infection may induce serious morbidity and mortality, especially in immunocompromised patients and transplant recipients [3]. Similarly, AdVs

\footnotetext{
* Correspondence: zlshi@wh.iov.cn

${ }^{1}$ Key Laboratory of Special Pathogens and Center for Emerging Infectious

Diseases, Wuhan Institute of Virology, Chinese Academy of Sciences, Wuhan, China

${ }^{3}$ University of Chinese Academy of Sciences, Beijing, China

Full list of author information is available at the end of the article
}

have been associated with diarrhea, acute respiratory illness, pneumonia, and hepatitis in captive non-human primates [4-6]. In 2009, an outbreak of AdV-infection led to $83 \%$ fatality in titi monkeys of the genus Callicebus, a group of New World monkeys [6]. Notably, neutralizing antibodies against this titi monkey mastadenovirus were detected in two human individuals, indicating the potential for zoonotic transmission. Several other cases of potential AdV transmission between humans and non-human primates have also been documented recently [7-9].

To date, at least 40 distinct types of simian adenoviruses (SAdVs) have been reported. Most of them infect captive great apes and members of the subfamily Cercopithecinae $[10,11]$. SAdVs that infect great apes are closely related to types that infect humans, which belong to the species Human mastadenovirus $A$ to Human mastadenovirus $F$ (HAdV-A-F) (primarily B, C, and E) [8, 11]; those infecting the Cercopithecinae members have been classified into 


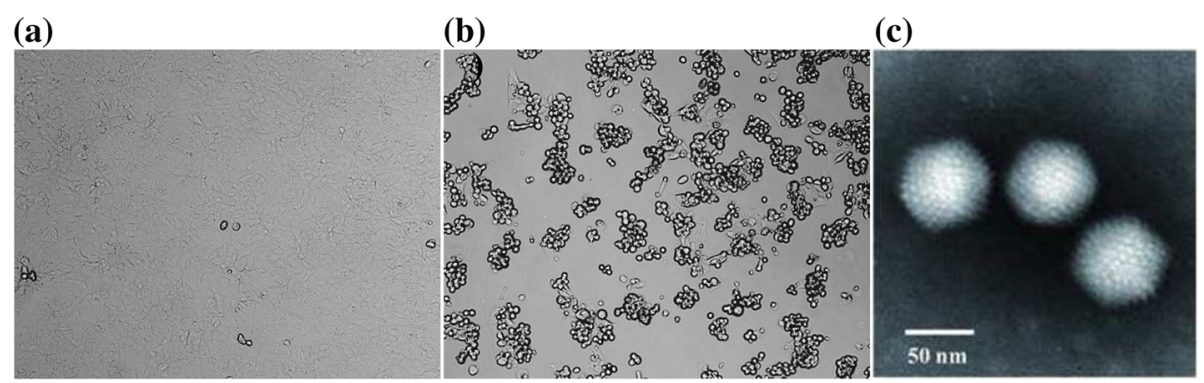

Fig. 1 Isolation of a simian adenovirus. a Uninfected Vero E6 cells. b Infected Vero E6 cells (second passage) inoculated with an adenovirus-positive sample at $72 \mathrm{~h}$ post infection. $\mathbf{c}$ Electron microscopy of purified adenoviral particles

HAdV-G, species Simian mastadenovirus A (SAdV-A), and the recently proposed species SAdV-B-H $[1,12,13]$. It should be noted that the group of Old World monkeys (OWMs) comprises two subfamilies, the Cercopithecinae and the Colobinae; however, knowledge of AdVs from the latter sub-group remains quite limited. Only a few short sequences of AdV DNA have been reported in this subfamily [11].

Golden snub-nosed monkeys (Rhinopithecus roxellana) living in Shennongjia Nature Reserve (SNR) in Hubei, China, are an endangered species belonging to the subfamily Colobinae [14]. Visitors to SNR have close contact with monkeys, which are fed by animal nurses in the reserve area; this raises the possibility of viral transmission between humans and monkeys. However, despite the extensive efforts to protect these monkeys from being endangered, viruses infecting these animals are poorly studied.

In this study, we conducted a surveillance of viral infections in 59 faecal samples from $R$. roxellana collected from SNR in 2014. Pan-PCR analysis was performed to detect the presence of AdVs, coronaviruses, enteroviruses, mammalian reoviruses, and rhinoviruses [15-17]. Except the 3 samples that tested positive for AdV, all samples were negative for the tested viruses. The virus strain in the 3 positive samples shared $100 \%$ nucleotide identity, based on the 261-bp DNA polymerase gene sequences. One of them was successfully isolated and cultured in Vero E6 cells (Fig. 1) and its full-length genome was sequenced. Following the order of viruses isolated in our laboratory at Wuhan Institute of Virology (WIV), we tentatively named this isolate as simian adenovirus WIV19 (SAdV WIV19).

The WIV19 genome comprises 33,562 bp, including 22.10\% A, 28.31\% C, 27.92\% G, and 21.67\% T (GenBank accession number KX505867) (Fig. 2). Its 95-bp extreme ends are inversely repeated, and start with the conserved motif CATCATCAAT [18]. The genome was predicted to encode 34 proteins and a virus-associated RNA (Fig. 2). Although some primate AdVs are known to encode two fibre genes, only one copy was identified in the genome of WIV19. Similarly, a single gene of noncoding virus-associated RNA was identified between the second exon of pTP and the 52K gene. Except for the absence of the E3 $12.5 \mathrm{~K}$ gene, organization of the WIV19 genome was identical to that of SAdV-A and G isolates infecting OWMs.

The putative gene products of the WIV19 genome displayed 22-91\% amino acid (aa) identities to their closest homologues encoded by members of SAdV-A (Additional file 1: Table S1). Most genes displayed $>85 \%$ aa identity with those encoding structural proteins, including pIIIa, penton base, pVII, pVI, pVIII, and hexon. By contrast, fibre protein showed only $32 \%$ aa identity to its closest homologue, which may suggest distinct infectivity in vivo due to its critical role in host cell binding [19]. DNA

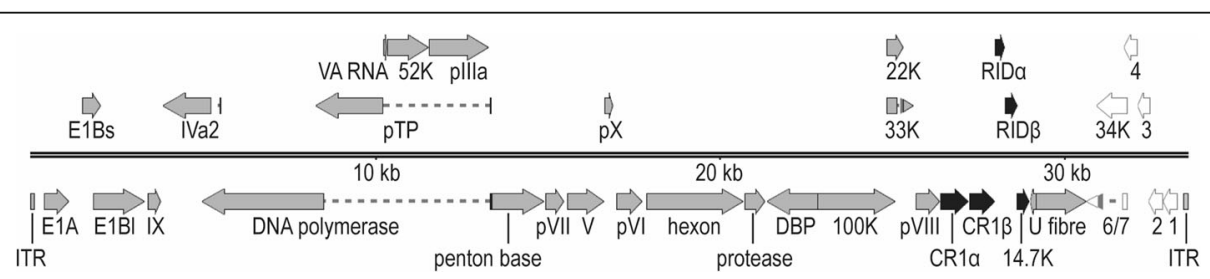

Fig. 2 Genomic characterization of SAdV-WIV19. The genome of SAdV-WIV19 is represented by the double line, with the scale indicated in the center. Viral genes and ITR sequences are shown as arrows and rectangles, respectively. Coding regions linked with broken lines indicate predicted spliced transcripts. The E3 and E4 genes are shown in black and white, respectively. With the exception of the 34K gene, the E4 genes are indicated by numbers of the corresponding open reading frames. Note that the genomic organization of WIV19 is similar to that shared by SAdV-A and G members, except that WIV19 lacks the E3 12.5K gene 

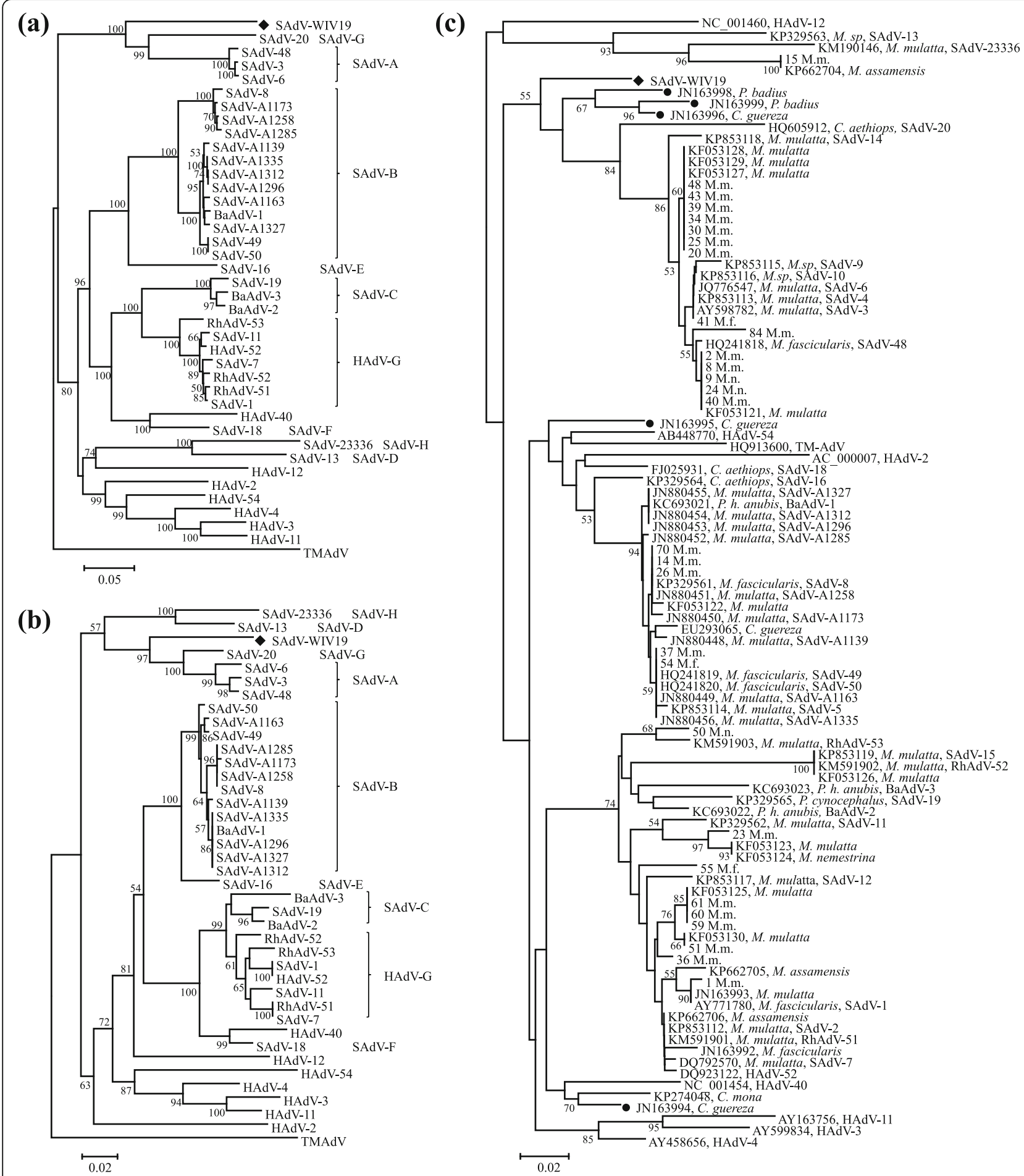

Fig. 3 Phylogenetic analysis based on the full-length amino acid sequences of DNA polymerase (a) and penton base (b) and partial nucleotide sequences of the hexon genes (c). Diamonds indicate the virus isolated in this study. Dots indicate adenoviruses from the subfamily Colobinae. HAdV, human adenovirus; SAdV, simian adenovirus; TMAdV, titi monkey adenovirus. In the partial hexon tree, adenoviruses infecting Old World monkeys are indicated by the GenBank accession numbers, followed by host species and type names (selected). "XX M.f.," "XX M.m.." and "XX M.n." (XX = sample number) indicate Chinese strains identified by Banyai, K., et al.; and M.m., M.f., and M.n. indicate the host species of Maccaca mulatta, M. fascicularis, and M. nemestrina, respectively [25]. C. mona, Cercopithecus mona; C. aethiops, Chlorocebus aethiops; C. guereza, Colobus guereza; P. cynocephalus, Papio cynocephalus; P. h. anubis, Papio hamadryas anubis; P. badius, Piliocolobus badius. The phylogenetic trees were constructed using the program MEGA, version 6.0, using the neighbor-joining method with 1,000 bootstrap replicates. Percentage bootstrap values $>50$ are indicated at the nodes. Scale bars indicate evolutionary distance 
polymerase is usually used for classification of AdV [1]. Based on analysis of sequences of this gene, WIV19 displayed the highest aa identity (77\%) to SAdV-3, suggesting that it is a distinct species.

Comparison of protein sequence similarities suggested that WIV19 is distantly related to OWM AdVs. Phylogenetic trees were constructed for better evaluation of the evolutionary relationship between WIV19 and the reported AdVs. Consistently, WIV19 formed a separate branch closer to a common ancestor of SAdV-A and G based on analyses of both DNA polymerase and penton base sequences (Fig. 3a, b). When we used the partial hexon sequences available in all OWM types, a common ancestor of three Colobinae types (JN163998, JN163999, and JN163996) was identified as the nearest neighbor to WIV19 (Fig. 3c). These observations suggested coevolution between WIV19 and golden snub-nosed monkeys.

Homologous recombination has been demonstrated to be an important evolutionary mechanism for AdVs, especially for HAdV-D members [20]. However, no signals of recombination were identified in the WIV19 genome, using RPD4 and SimPlot software [21, 22].

For investigating the antibody prevalence against the AdV infection, virus neutralization assay was performed using archived serum samples collected from 8 golden snub-nosed monkeys in SNR [23]. As a result, crossneutralizing antibodies against WIV19 were identified in all the 8 samples at titers ranging from 40 to 160 .

The potential host range of WIV19 was evaluated using cell infectivity assays. Seven cell lines were tested: A549 (human lung, ATCC CRM-CCL-185), HEK-293 T (human embryonic kidney, ATCC CRL-11268), NCI-H292 (human lung, ATCC CRL-1848), LLC-MK2 (Macaca mulatta kidney, ATCC CCL-7), MsIn (Miniopterus schreibersi intestine), RIKi (Rousettus leschenaulti kidney), and RsKi (Rhinolophus sinicus kidney) [24]. Cytopathic effects were observed in LLC-MK2, RsKi, and all three human cell lines, indicating that WIV19 has a wide host range.

In conclusion, we described here the first isolation and characterization of a replication-competent AdV from monkeys in the subfamily Colobinae. The cumulative prevalence of AdV in endangered golden snub-nosed monkeys from SNR was found to be $5.1 \%$. The present isolate from these monkeys is distantly related to known AdV types, and likely represents a novel species in the genus Mastadenovirus. Its ability to infect diverse cell lines, including those of human origin, highlights the potential risk of new and emerging infections in tourists visiting SNR. Result from the virus neutralization assay suggested that the infections of WIV19 may be highly prevalent in these monkeys. Further studies are required to determine its host range and role in causing disease in both golden snub-nosed monkeys and humans. Long-term surveillance of viral prevalence in this region should be conducted in the future.

\section{Additional file}

Additional file 1: Table S1. Predicted genes of WIV19 and comparison to those of known adenoviruses. (DOCX $20 \mathrm{~kb}$ )

\section{Abbreviations \\ AdVs: Adenoviruses; HAdV: Human mastadenovirus; OWMs: Old World monkeys; SAdV: Simian adenovirus or mastadenovirus; SNR: Shennongjia Nature Reserve; WIV: Wuhan Institute of Virology}

\section{Acknowledgements}

This work was jointly funded by the National Natural Science Foundation of China (81290341) and China Mega-Project for Infectious Disease (2014ZX10004001-003) and the Scientific and Technological Basis Special Project (2013FY113500) from the Ministry of Science and Technology of the People's Republic of China.

\section{Availability of data and material}

The assembled sequence data were uploaded to the GenBank database (http://www.ncbi.nlm.nih.gov/nuccore) at the accession number of KX505867.

\section{Authors' contributions}

BT, LJW, YSL, YL, GXY, JC, GC, HZW, and ZLS collected samples. XLY, BL, and WZ performed the PCR assays. BT performed the other experiments and analyzed the data. BT and ZLS conceived of the study and drafted the manuscript. All authors read and approved the final manuscript.

\section{Competing interests}

The authors declare that they have no competing interests.

\section{Consent for publication}

Not applicable.

Ethics approval and consent to participate Not applicable.

\section{Author details \\ ${ }^{1}$ Key Laboratory of Special Pathogens and Center for Emerging Infectious Diseases, Wuhan Institute of Virology, Chinese Academy of Sciences, Wuhan, China. ${ }^{2}$ Monitoring Center of Wildlife Diseases and Resource of Hubei Province, Wuhan, China. ${ }^{3}$ University of Chinese Academy of Sciences, Beijing, China. ${ }^{4}$ Present Address: Key Laboratory of Special Pathogens and Biosafety, Wuhan Institute of Virology, Chinese Academy of Sciences, Wuhan 430071, China.}

Received: 1 August 2016 Accepted: 10 November 2016 Published online: 25 November 2016

\section{References}

1. Harrach B, Benkö M, Both GW, Brown M, Davison AJ, Echavarría M, Hess M, Jones MS, Kajon A, Lehmkuhl HD, et al. Family Adenoviridae. In: King AMQ, Adams MJ, Carstens EB, Lefkowitz EJ, editors. Virus Taxonomy: Classification and Nomenclature of Viruses: Ninth Report of the International Committee on Taxonomy of Viruses. San Diego: Elsevier; 2011. p. 125-41.

2. Ghebremedhin B. Human adenovirus: Viral pathogen with increasing importance. Eur J Microbiol Immunol (Bp). 2014;4:26-33.

3. Echavarria M. Adenoviruses in immunocompromised hosts. Clin Microbiol Rev. 2008;21:704-15

4. Chiu CY, Yagi S, Lu X, Yu G, Chen EC, Liu M, Dick Jr EJ, Carey KD, Erdman DD, Leland MM, Patterson JL. A novel adenovirus species associated with an acute respiratory outbreak in a baboon colony and evidence of coincident human infection. MBio. 2013:4:e00084

5. Wang Y, Tu X, Humphrey C, McClure H, Jiang X, Qin C, Glass Rl, Jiang B. Detection of viral agents in fecal specimens of monkeys with diarrhea. J Med Primatol. 2007;36:101-7.

6. Chen EC, Yagi S, Kelly KR, Mendoza SP, Tarara RP, Canfield DR, Maninger N, Rosenthal A, Spinner A, Bales KL, et al. Cross-species transmission of a novel adenovirus associated with a fulminant pneumonia outbreak in a new world monkey colony. PLoS Pathog. 2011;7:e1002155. 
7. Jones 2nd MS, Harrach B, Ganac RD, Gozum MM, Dela Cruz WP, Riedel B, Pan C, Delwart EL, Schnurr DP. New adenovirus species found in a patient presenting with gastroenteritis. J Virol. 2007;81:5978-84.

8. Hoppe E, Pauly M, Gillespie TR, Akoua-Koffi C, Hohmann G, Fruth B, Karhemere S, Madinda NF, Mugisha L, Muyembe JJ, et al. Multiple cross-species transmission events of human adenoviruses (HAdV) during Hominine evolution. Mol Biol Evol. 2015;32:2072-84.

9. Roy S, Vandenberghe LH, Kryazhimskiy S, Grant R, Calcedo R, Yuan X, Keough M, Sandhu A, Wang Q, Medina-Jaszek CA, et al. Isolation and characterization of adenoviruses persistently shed from the gastrointestinal tract of non-human primates. PLoS Pathog. 2009:5:e1000503.

10. Seimon TA, Olson SH, Lee K, Rosen G, Ondzie A, Cameron K, Reed P, Anthony SJ, Joly DO, Karesh WB, et al. Adenovirus and herpesvirus diversity in free-ranging great apes in the Sangha region of the Republic Of Congo. PLoS One. 2015:10:e0118543.

11. Wevers D, Metzger S, Babweteera F, Bieberbach M, Boesch C, Cameron K, Couacy-Hymann E, Cranfield M, Gray M, Harris LA, et al. Novel adenoviruses in wild primates: a high level of genetic diversity and evidence of zoonotic transmissions. J Virol. 2011;85:10774-84.

12. Panto L, Podgorski II, Janoska M, Marko O, Harrach B. Taxonomy proposal for Old World monkey adenoviruses: characterisation of several non-human, non-ape primate adenovirus lineages. Arch Virol. 2015;160:3165-77.

13. Podgorski II, Panto L, Papp T, Harrach B, Benko M. Genome analysis of four Old World monkey adenoviruses supports the proposed species classification of primate adenoviruses and reveals signs of possible homologous recombination. J Gen Virol. 2016:97:1604-14.

14. Yongcheng L, Richardson M. Rhinopithecus roxellana, The IUCN Red List of Threatened Species 2008: eT19596A8985735. 2008.

15. Li Y, Ge X, Zhang H, Zhou P, Zhu Y, Zhang Y, Yuan J, Wang LF, Shi Z. Host range, prevalence, and genetic diversity of adenoviruses in bats. J Virol. 2010;84:3889-97.

16. Coiras MT, Aguilar JC, Garcia ML, Casas I, Perez-Brena P. Simultaneous detection of fourteen respiratory viruses in clinical specimens by two multiplex reverse transcription nested-PCR assays. J Med Virol. 2004;72:484-95.

17. Yang XL, Tan B, Wang B, Li W, Wang N, Luo CM, Wang MN, Zhang W, Li B, Peng $C$, et al. Isolation and identification of bat viruses closely related to human, porcine, and mink orthoreoviruses. J Gen Virol. 2015;96:3525-31.

18. Yang Z, Zhu Z, Tang L, Wang L, Tan X, Yu P, Zhang Y, Tian X, Wang J, Li D, Xu W. Genomic analyses of recombinant adenovirus type 11a in China. J Clin Microbiol. 2009;47:3082-90.

19. San Martin C, Burnett RM. Structural studies on adenoviruses. Curr Top Microbiol Immunol. 2003;272:57-94.

20. Singh G, Robinson CM, Dehghan S, Jones MS, Dyer DW, Seto D, Chodosh J. Homologous recombination in E3 genes of human adenovirus species D. J Virol. 2013;87:12481-8.

21. Lole KS, Bollinger RC, Paranjape RS, Gadkari D, Kulkarni SS, Novak NG, Ingersoll R, Sheppard HW, Ray SC. Full-length human immunodeficiency virus type 1 genomes from subtype C-infected seroconverters in India, with evidence of intersubtype recombination. J Virol. 1999;73:152-60.

22. Martin DP, Williamson C, Posada D. RDP2: recombination detection and analysis from sequence alignments. Bioinformatics. 2005;21:260-2.

23. Ge XY, Li JL, Yang XL, Chmura AA, Zhu G, Epstein JH, Mazet JK, Hu B, Zhang W, Peng $C$, et al. Isolation and characterization of a bat SARS-like coronavirus that uses the ACE2 receptor. Nature. 2013;503:535-8.

24. Tan B, Yang XL, Ge XY, Peng C, Zhang YZ, Zhang LB, Shi ZL. Novel bat adenoviruses with an extremely large E3 gene. J Gen Virol. 2016;97(7):1625-35.

25. Banyai K, Esona MD, Liu A, Wang Y, Tu X, Jiang B. Molecular detection of novel adenoviruses in fecal specimens of captive monkeys with diarrhea in China. Vet Microbiol. 2010;142:416-9.

\section{Submit your next manuscript to BioMed Central and we will help you at every step:}

- We accept pre-submission inquiries

- Our selector tool helps you to find the most relevant journal

- We provide round the clock customer support

- Convenient online submission

- Thorough peer review

- Inclusion in PubMed and all major indexing services

- Maximum visibility for your research

Submit your manuscript at www.biomedcentral.com/submit
Biomed Central 